\title{
Belgeo
}

Revue belge de géographie

$3 \mid 2019$

Les petites villes européennes comme enjeu d'équité territoriale/Small European cities as stakes for territorial equity

\section{Quelles dynamiques pour les petites villes en Europe ? Une étude comparée Saxe-Bretagne (1990-2015)}

What dynamics for small towns in Europe? A comparative study between Saxony and Brittany (1990-2015)

Guy Baudelle, Joachim Burdack et Louis-Thibault Buron

\section{(2) OpenEdition}

Journals

Édition électronique

URL : http://journals.openedition.org/belgeo/34305

DOI : $10.4000 /$ belgeo.34305

ISSN : 2294-9135

Éditeur :

National Committee of Geography of Belgium, Société Royale Belge de Géographie

Référence électronique

Guy Baudelle, Joachim Burdack et Louis-Thibault Buron, «Quelles dynamiques pour les petites villes en Europe? Une étude comparée Saxe-Bretagne (1990-2015) », Belgeo [En ligne], 3 | 2019, mis en ligne le 17 mai 2019, consulté le 13 février 2020. URL : http://journals.openedition.org/belgeo/34305 ; DOI : 10.4000/belgeo.34305

Ce document a été généré automatiquement le 13 février 2020.

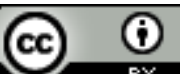

Belgeo est mis à disposition selon les termes de la licence Creative Commons Attribution 4.0 International. 


\section{Quelles dynamiques pour les petites villes en Europe ? Une étude comparée Saxe-Bretagne (1990-2015)}

What dynamics for small towns in Europe? A comparative study between Saxony and Brittany (1990-2015)

Guy Baudelle, Joachim Burdack et Louis-Thibault Buron

\section{Introduction}

1 Les déterminants généraux des trajectoires démographiques des petites villes européennes sont relativement mal connus en raison du faible intérêt de la recherche pour cette question et de la grande disparité d'évolution observée. C'est pourquoi l'article propose une typologie d'évolution à l'échelle régionale. On a choisi d'étudier les petites villes de Saxe (Allemagne orientale) et de Bretagne (France). Cette comparaison trouve sa source dans le jumelage de ces deux régions qui ont développé des coopérations scientifiques. L'analyse de cas aussi contrastés pourrait étonner mais l'identification des facteurs d'évolution des villes européennes suppose précisément de retenir des terrains représentatifs de leur diversité (Bell, Jayne, 2009; Baron et al., 2010).

2 Compte tenu de la grande hétérogénéité d'évolution des petites villes en Europe et de logiques apparemment plus régionales que propres à un niveau hiérarchique, on a posé en hypothèse que leurs trajectoires reflètent avant tout les tendances démographiques de leur région, ce que l'étude confirme sans exclure de fortes variations. On a classé ces villes en plusieurs types en fonction de leur rythme d'évolution et des facteurs en rendant compte. Les variations internes s'expliquent avant tout par la proximité d'agglomérations dynamiques ( $\mathrm{du}$ fait de la portée et de l'intensité de l'onde de 
périurbanisation) et par leur base économique initiale tandis que l'économie résidentielle intervient surtout en Bretagne.

On justifie d'abord la comparaison entre deux régions qu'apparemment tout oppose en abordant les problèmes méthodologiques qu'elle soulève (I) avant de présenter successivement la Saxe qui se caractérise par cinq profils d'évolution (II), puis la Bretagne qui présente également six types contrastés (III). Cette comparaison régionale permet de confirmer en conclusion certains faits connus mais dément toute crise générale de cette strate urbaine en soulignant néanmoins leur dépendance aux dynamiques sociodémographiques des autres agglomérations et de leur région.

\section{Une comparaison régionale}

\section{Une strate urbaine mal connue}

4 Selon Antoine Delmas et Dominique Royoux (2019), « les petites villes incarnent une réalité méconnue de l'urbanisation » en raison d'un manque de travaux (Bell, Jayne, 2009 ; Manfred, 2015 ; Steinführer et al., 2016) sur «ces villes dont on ne parle pas » (Authier, Bidou-Zachariasen, 2017), partiellement dû à un moindre intérêt pour les espaces ruraux auxquels elles sont associées (Carlow, 2017), malgré des recherches pionnières (Grötzbach, 1963 ; Kayser et al., 1972 ; Laborie, 1979).

On observe cependant depuis une douzaine d'années un regain d'intérêt scientifique pour ce thème, qui se poursuit (Châtel, Moriconi-Ebrard, 2016-2017 ; Batunova, Gunko, 2017 ; Varna et al., 2018). Les organes en charge de l'aménagement du territoire ne sont pas en reste (BMVBS, 2013 ; Maretzke, 2018 ; PUCA, 2018).

6 Cette lacune surprend vu l'extrême diversité des trajectoires démographiques des petites villes européennes (Fertner et al., 2015 ; Wolff, 2018). Le profil des petites villes allemandes est ainsi très contrasté (BMVBS, 2013 ; Manfred, 2015 ; Maretzke, 2018) tout comme leurs perspectives (Lüttich, 2017). L'évolution des villes baltiques (Grundfelder et al., 2018), polonaises (Kamińska, Mularczyk, 2014) ou hongroises (Gábor et al., 2015) confirme cette hétérogénéité (Delmas, Royoux, 2019), contredisant leur prétendue dévitalisation générale. La proportion de villes européennes en déclin augmente même avec leur taille (Wolff, Wiechmann, 2017)!

\section{Deux régions apparemment opposées mais toutes deux périphériques}

7 Très différentes sur le plan démographique et économique, Saxe et Bretagne partagent néanmoins le même statut de périphérie. La Bretagne n'occupait en 2013 que le $12^{\mathrm{e}}$ rang (sur 21) pour le PIB par tête ( $26636 €$ ) et le $16^{\mathrm{e}}$ ex-aequo pour le salaire net annuel du secteur concurrentiel (84,7\% de la moyenne de 2010). En Saxe en 2013, les valeurs étaient de $24226 €$ pour le PIB/h. (72,6\% de la moyenne fédérale) et de $25492 €$ pour le salaire annuel par employé ( $82 \%$ de la moyenne).

La comparaison entre les deux pays est rendue possible par l'identité des seuils statistiques officiels définissant la petite ville: côté français une unité urbaine l'agglomération morphologique - de 2000 à 20000 habitants agglomérés ${ }^{1}$, la ville ou la 
commune côté allemand. Les tableaux 1 et 2 indiquent le poids de ces villes dans leur pays.

Tableau 1. Les petites villes en Allemagne (2017).

\begin{tabular}{lccc}
\hline Classe de taille & $\begin{array}{c}\text { Nombre de petites } \\
\text { villes }\end{array}$ & $\begin{array}{c}\text { Population } \\
\text { (en milliers) }\end{array}$ & $\begin{array}{c}\text { Part de la population } \\
\text { nationale (en \%) }\end{array}$ \\
\hline $2000-5000$ & 326 & 1166 & 1,4 \\
$5000-10000$ & 484 & 3567 & 4,4 \\
$10000-20000$ & 519 & 7502 & 9,2 \\
Total & 1329 & 12235 & 15 \\
\hline
\end{tabular}

Source : Destatis

Tableau 2. Les petites villes en France (2015).

\begin{tabular}{lccc}
\hline Classe de taille & $\begin{array}{c}\text { Nombre de petites } \\
\text { villes }\end{array}$ & $\begin{array}{c}\text { Population } \\
\text { (en milliers) }\end{array}$ & $\begin{array}{c}\text { Part de la population de } \\
\text { métropole (en \%) }\end{array}$ \\
\hline $2000-5000$ & 1163 & 3960 & 6,2 \\
$5000-10000$ & 578 & 3973 & 6,2 \\
$10000-20000$ & 236 & 3278 & 5,1 \\
Total & 1977 & 11211 & 17,4 \\
\hline
\end{tabular}

Source : INSEE

9 Le nombre de petites villes est de surcroît analogue dans les deux régions (164 en Bretagne, 138 en Saxe) et leur poids similaire : environ un million d'habitants dans les deux cas, soit $27,1 \%$ de la population en Saxe et $31,6 \%$ en Bretagne.

10 Les deux régions ont un poids démographique équivalent avec 3,3 millions de Bretons pour 4 millions de Saxons (fin 2017). En revanche, la chronologie de leur évolution démographique est complètement asymétrique puisque la Saxe a vu sa population augmenter (de 4,5 à 5,7 millions de 1905 à 1950) quand elle déclinait en Bretagne (de 2,6 à 2,3 millions de 1901 à 1954) et inversement depuis 1950 . Avec une baisse de $18 \%$, la Saxe a perdu près d'un million d'habitants entre 1990 et 2017, régressant de 4,91 à 4 millions à peine. De son côté, la population bretonne a crû de près de 17 \% entre 1990 et 2017, s'élevant de moins de 2,8 à près de 3,3 millions. Les deux régions ont donc connu une évolution en ciseau. Même contraste économique: la Saxe a connu des restructurations considérables depuis la réunification alors que la Bretagne a vécu une forte expansion.

\section{Questions méthodologiques}

11 D'une manière générale, la quête de facteurs explicatifs peut s'appuyer sur la comparaison de régions à la fois homothétiques et divergentes (Baron et al., 2010). Les études comparées entre pays posent cependant classiquement d'importantes questions.

La première porte sur la définition de la petite ville. La démographie reste le principal critère d'identification (Demazière, 2017), a fortiori commode pour repérer les trajectoires d'une même strate et en cerner les raisons. Les seuils retenus, outre leur valeur officielle, sont les plus couramment choisis par les chercheurs (Taulelle, 2010; Santamaria, 2012 ; Delmas, Royoux, 2019). Sur la base des services et de l'emploi, seule 
une faible partie des petites unités urbaines ferait figure de petit pôle (selon le vocable de l'INSEE) ou de centre de base (Grundzentrum ou Unterzentrum selon la terminologie allemande des lieux centraux).

13 La seconde difficulté concerne la disparité de taille entre communes: celles de Saxe étaient en 2015 deux fois plus étendues $\left(42 \mathrm{~km}^{2}\right)$ que leurs homologues bretonnes (21 $\mathrm{km}^{2}$ ) du fait notamment des nombreuses fusions intervenues en Allemagne dans les années 1990 et 2000. La plus grande superficie communale dans l'Ouest français d'habitat dispersé par rapport à la moyenne nationale (moins de $15 \mathrm{~km}^{2}$ ) atténue cependant l'inconvénient de la comparaison entre territoires aux trames communales distinctes.

L'écart de population entre petites villes est d'ailleurs faible ( $5500 \mathrm{~h}$. en Saxe contre 6000 en Bretagne) car l'unité urbaine de l'INSEE peut en réalité englober plusieurs communes (formant alors une agglomération multicommunale), ce qui rend la comparaison plus pertinente.

15 Enfin, quatre petites villes ont statistiquement disparu en Saxe suite à leur fusion depuis 1990 et une quinzaine d'autres communes en ont incorporé d'autres. Nous les avons considérées dans leurs limites de 2015.

\section{Les petites villes allemandes : évolution et typologie}

\section{L'évolution récente des petites villes de Saxe}

La trame médiévale des petites villes de Saxe a été complétée par les villes minières des Monts Métallifères (fin $\mathrm{XV}^{\mathrm{e}}$-début $\mathrm{XVI}^{\mathrm{e}}$ ). Très peu de petites villes sont apparues ultérieurement.

Le poids économique de ces 138 petites villes a sensiblement diminué depuis le processus de transformation d'après 1990, nombre d'entreprises d'Etat (VEB) qui formaient la base économique des petites villes n'étant plus compétitives dans une économie de marché. De nombreuses petites villes de l'ex-RDA n'ont pas seulement souffert de désindustrialisation mais aussi de désadministration et de démilitarisation du fait des pertes d'emplois provoquées par la disparition de certaines fonctions (Hannemann, 2004), souvent au profit des centres supérieurs (Lentz, 2004). Elles ont presque toutes perdu de la population depuis la réunification (figure 1). Leur évolution démographique a été encore plus médiocre qu'en Saxe en général puisque près des trois quarts d'entre elles ont connu une baisse plus prononcée que la moyenne régionale $(-16,9 \%)$. Dix seulement, presque toutes périurbaines, ont vu leur population croître depuis 1990. 
Figure 1. Les petites villes de Saxe : population et évolution démographique (1990-2015).

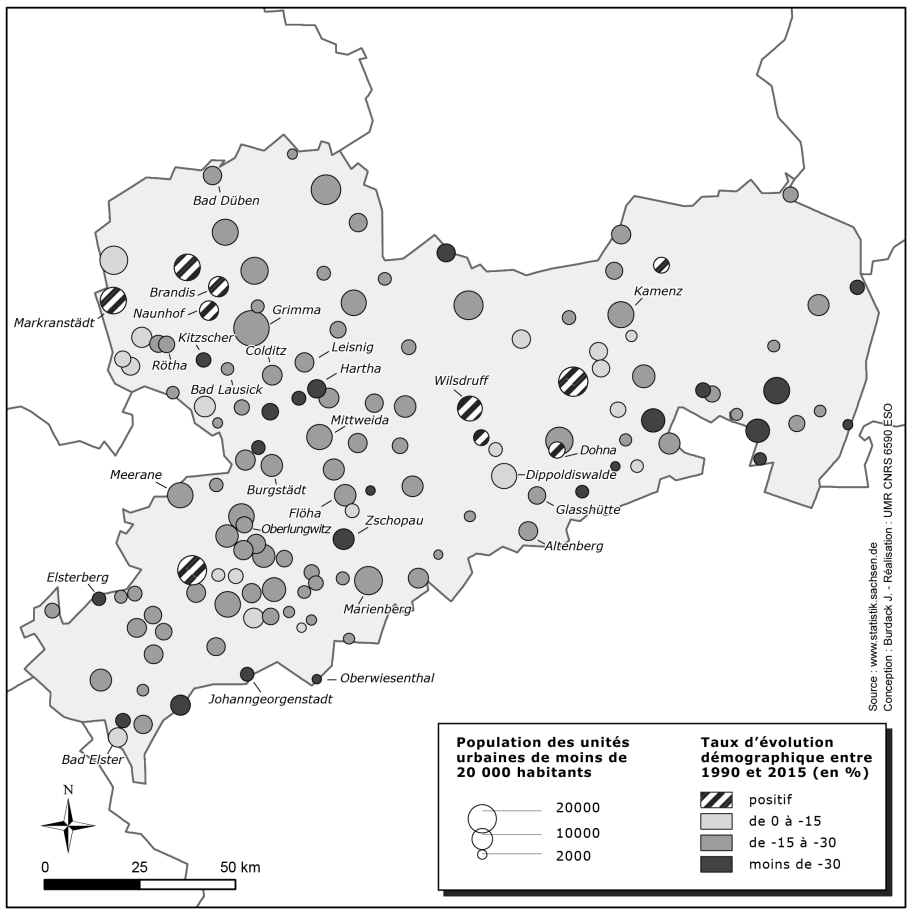

De nombreuses petites villes saxonnes avaient fondé leur économie sur l'artisanat et l'industrie depuis le XVIII ${ }^{\mathrm{e}}$ ou XIX ${ }^{\mathrm{e}} \mathrm{s}$. à l'instar de l'ancien district marshallien textile autour de Chemnitz avec la ganterie (Burgstädt), la filature du coton (Flöha, Mittweida) ou la bonneterie (Oberlungwitz). Quelques localités du Vogtland étaient spécialisées dans les instruments de musique. Bon nombre de ces spécialités traditionnelles ont perduré et se sont même renforcées au temps de la RDA. Alors que la plupart des grandes villes comme Chemnitz et Dresde avaient souffert de graves destructions de guerre, beaucoup de petites villes y avaient échappé, conservant une grande partie de leur patrimoine. Sous le communisme, l'investissement a toutefois privilégié les grandes villes. Les petites qui n'étaient pas chef-lieu d'arrondissement se sont trouvées dans une situation difficile, souffrant de la dégradation du bâti et des infrastructures. Leur déclin démographique a bien souvent commencé avant la réunification.

\section{Diversité des trajectoires des petites villes saxonnes : cinq profils démographiques}

Toutes ces villes ont été durement frappées par la transformation économique et sociale au début des années 1990, en particulier les centres industriels contraints de réduire leurs effectifs, d'autant que de nombreuses fonctions annexes étaient intégrées aux entreprises à l'époque de la RDA. On peut distinguer cinq types d'évolution depuis 1990 en fonction de la trajectoire démographique et du profil économique de départ (tableau 3). 
Tableau 3. Typologie des petites villes saxonnes d'après leur évolution démographique et de leur profil économique (2015).

\begin{tabular}{|l|c|c|c|c|}
\hline Type & Nombre & $\begin{array}{c}\text { Part des petites } \\
\text { villes }\end{array}$ & $\begin{array}{c}\text { Evolution de } \\
\text { l'emploi } \\
1998-2015\end{array}$ & $\begin{array}{c}\text { Evolution } \\
\text { démographique } \\
1990-2015\end{array}$ \\
\hline Petites villes désindustrialisées & 35 & $30 \%$ & $-23 \%$ & $-26 \%$ \\
\hline Petites centres réindustrialisés & 28 & $24 \%$ & $-9 \%$ & $-24 \%$ \\
\hline Petits pôles de services spécialisés & 17 & $15 \%$ & $-10 \%$ & $-23 \%$ \\
\hline Petits lieux centraux renforcés & 19 & $16 \%$ & $-10 \%$ & $-25 \%$ \\
\hline Petites localités périurbaines & 17 & $15 \%$ & $-2 \%$ & $+6 \%$ \\
\hline Ensemble des cinq types & $\mathbf{1 1 6}$ & $\mathbf{1 0 0} \%$ & $\mathbf{- 1 2} \%$ & $-\mathbf{2 0 , 9} \%$ \\
\hline $\begin{array}{l}\text { Petites villes non classées faute de } \\
\text { données suffisantes }\end{array}$ & 22 & & & $-20,5 \%$ \\
\hline Ensemble des petites villes & 138 & & & \\
\hline
\end{tabular}

Source : Statistisches Landesamt Sachsen

20 La petite ville désindustrialisée correspond à un type anciennement industrialisé où le secteur manufacturier ne joue plus qu'un rôle mineur. Ce profil se rencontre en particulier à l'ouest autour de Chemnitz/Zwickau et dans le Vogtland (figure 2).

Figure 2. La place du marché de Colditz, exemple de réaménagement d'une ville désindustrialisée, grâce aux programmes de rénovation urbaine (Stadterneuerung).

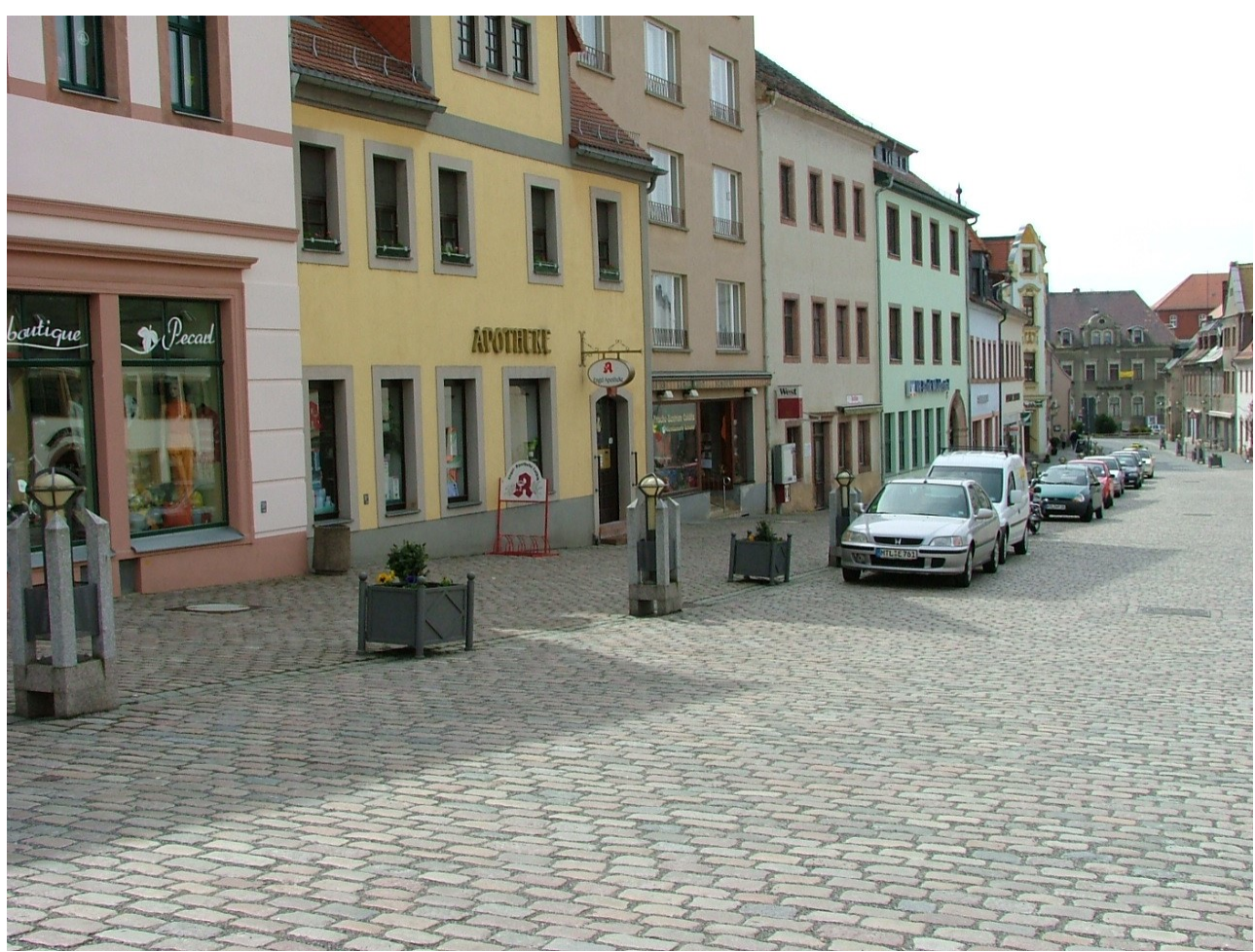

Source : ( ) J. Burdack

21 Le meilleur exemple de petit centre réindustrialisé est sans doute Glashütte (6 $800 \mathrm{~h}$.), héritier d'une grande tradition de fabrication de montres mécaniques. Les PME horlogères, présentes depuis le XIX et regroupées au sein de la VEB Glashütter 
Uhrenbetriebe (GUB) au temps de la RDA, fabriquent désormais des montres mécaniques haut de gamme.

Les petits pôles de services spécialisés se trouvent pour leur part dans des zones pittoresques attrayantes. Ce sont des destinations touristiques, notamment des stations thermales.

Certaines petites villes ont réussi à renforcer leur fonction de lieu central (au sens christallérien) pour les zones rurales environnantes, principalement dans le haut de la strate urbaine (plus de $10000 \mathrm{~h}$.) (figure 3).

Figure 3. Le centre-ville (Marktplatz) de Grimma, lieu central requalifié sur crédits de la Stadterneuerung.

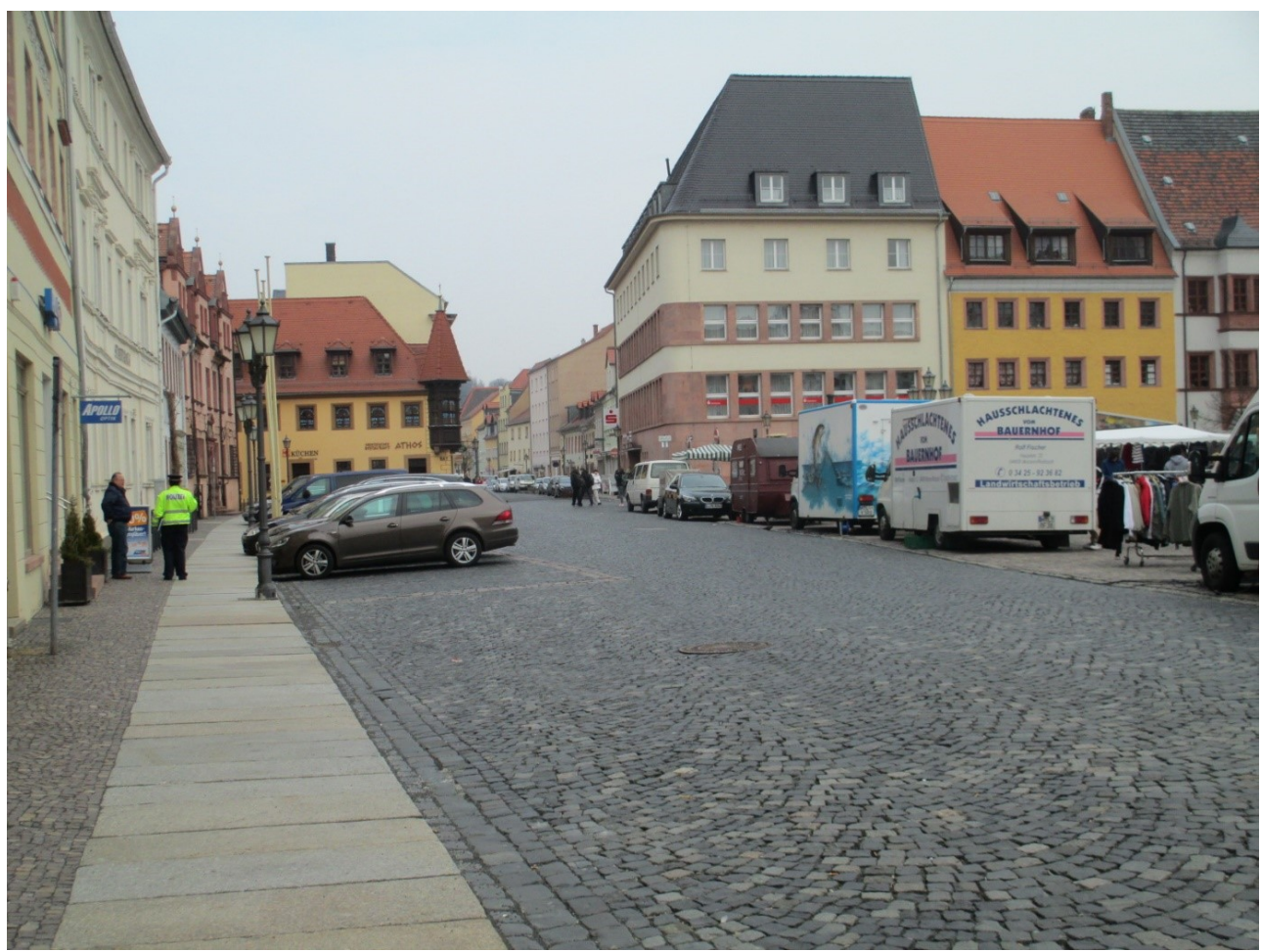

Source : ( c J. Burdack

petites localités périurbaines forment un type particulier à cause de leurs liens étroits avec des agglomérations plus grandes. Ce sont d'anciens centres ruraux dorénavant intégrés à la dynamique d'aires urbaines du fait du spectaculaire déversement résidentiel périurbain.

En dehors des couronnes périurbaines, on ne trouve qu'une seule petite ville dont la population a crû depuis la réunification. Le déficit démographique a été généralisé en dehors du périurbain jusqu’à 2005 (figure 4). 
Figure 4. Les petites villes saxonnes : évolution de la population par type de ville de 1990 à 2015 (\% annuel).

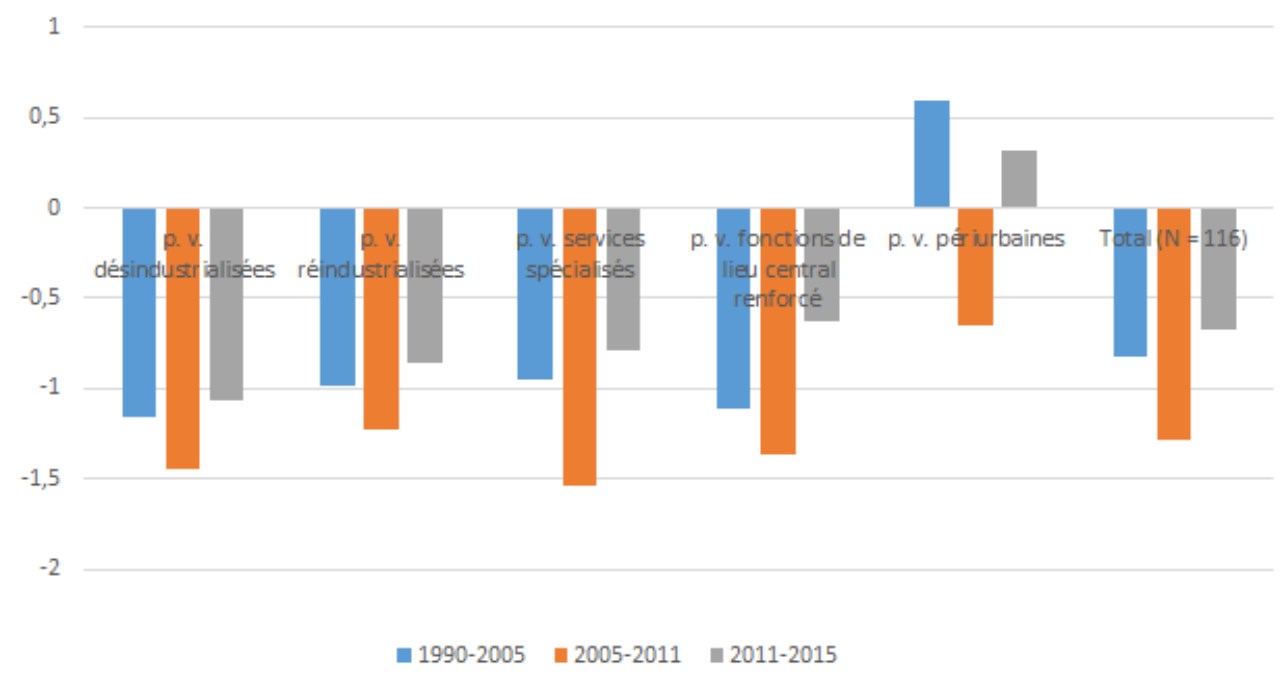

La dynamique de l'emploi a été plus favorable (figure 5). Les petites villes ayant renforcé leurs fonctions de lieu central ont connu une croissance substantielle, le taux d'emploi (pour 1000 habitants) en 2015 y étant supérieur (397) à la moyenne régionale (375) alors qu'il reste très faible (236) dans les petites villes désindustrialisées.

Figure 5. Les petites villes saxonnes : évolution de l'emploi par type de 1990 à 2015 (\% annuel).

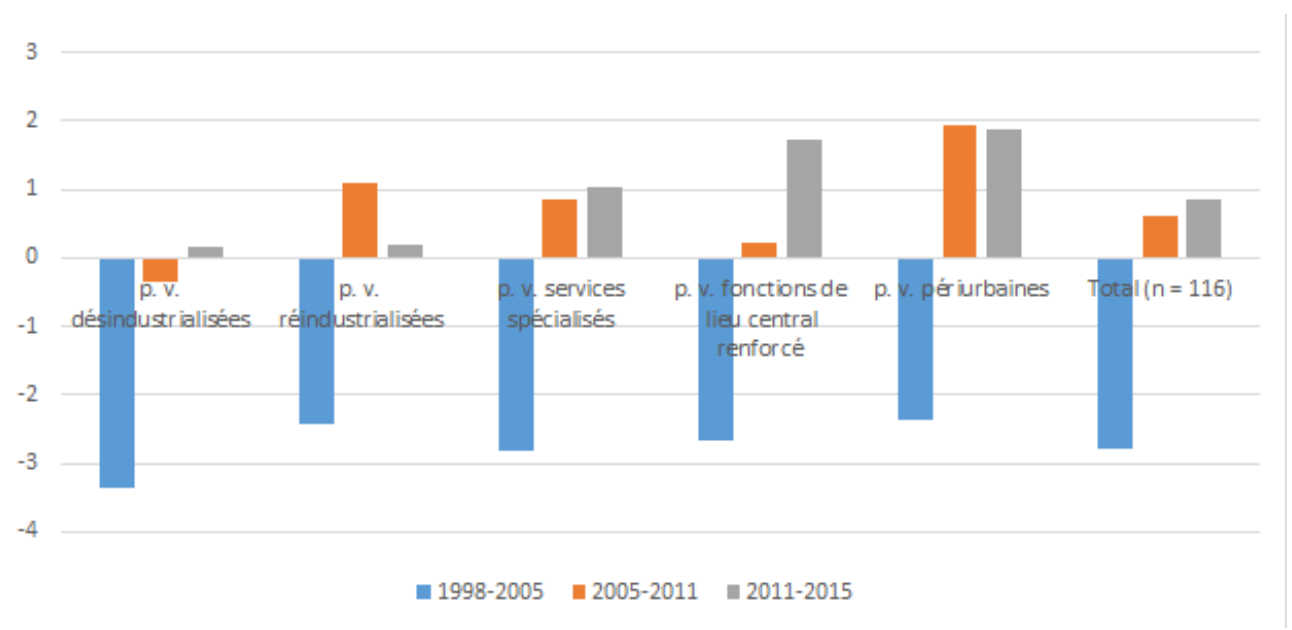

\section{Villes marginalisées vs. villes revitalisées}

Il est utile de compléter cette typologie par une autre basée sur les efforts de reconversion qui oppose les villes à base économique renouvelée ayant stabilisé leur trajectoire à celles ayant connu une spirale de déclin économique. Les deux extrêmes sont la petite ville revitalisée et la petite ville marginalisée.

Le concept de marginalisation (Peripherisierung) signale une perte de fonctions et de rayonnement par rapport à un centre dominant, avec un « processus d'interaction entre la faiblesse économique structurelle et les changements démographiques» (Barlösius, Neuer, 2008). Les petites villes marginalisées (ou périphériques) ont vu s'effondrer leur capital 
territorial (économique, environnemental, humain, social) défini comme l'ensemble de leur potentiel : réseaux sociaux traditionnels, savoir-faire ou identité régionale (Leber, Kunzmann, 2006). La marginalisation est en effet un processus multidimensionnel: économique (perte d'emplois, déficit d'innovation), sociodémographique (perte de capital humain par départ de populations jeunes et qualifiées), fonctionnelle (suppression des équipements : collège, poste, transports, etc.), sociale (manque de participation) et culturelle (image de marque négative: la marginalisation mentale, "dans les têtes») (Bose et Wirth, 2006). La petite ville marginalisée n'attire plus d'investissements en dehors de l'exploitation de matières premières. Le potentiel de participation passe sous le seuil critique nécessaire à des stratégies de développement endogène. Pessimisme et renoncement paralysent la population et empêchent la mobilisation des ressources. Le syndrome de la petite ville périphérique comporte donc une dimension objective (économique et démographique) et subjective (mentalités, capacités d'initiative et d'action collective).

L'analyse de ces deux dimensions est nécessaire : on doit étudier finement sur le terrain capital social, réseaux sociaux et images interne et externe (Borsig et al. 2010 ; Burdack, 2010). La marginalisation peut aussi se voir à l'aide d'indicateurs socio-économiques objectifs. Quand une ville, par exemple, perd environ le tiers de sa population en deux décennies, le fonctionnement de l'infrastructure sociale et technique ne peut plus être garanti que par des efforts d'adaptation considérables. Des problèmes de vacance immobilière, de fermetures d'écoles ou de budget peuvent difficilement être contrecarrés. Ajoutez-y une forte baisse de l'emploi et la marginalisation est évidente. Si on prend un recul démographique d'au moins un tiers, une diminution de l'emploi et une hausse du chômage comme indices, alors $5 \%$ environ des petites villes saxonnes sont exposées à la marginalisation (tableau 4).

Tableau 4. Les petites villes en fort déclin démographique (1990-2015).

\begin{tabular}{lccc}
\hline & 1990 & 2015 & Evolution en \% \\
\hline Johanngeorgenstadt & 9094 & 4135 & $-54,5$ \\
Oberwiesenthal & 4420 & 2194 & $-50,4$ \\
Seifhennersdorf & 6874 & 3817 & $-44,5$ \\
Königstein & 3625 & 2122 & $-41,5$ \\
Kitzscher & 8553 & 5034 & $-41,1$ \\
Wilthen & 8510 & 5091 & $-40,2$ \\
Ebersbach-Neugersdorf & 20394 & 12484 & $-38,8$ \\
Ostritz & 3867 & 2382 & $-38,4$ \\
\hline
\end{tabular}

Source : Statistisches Landesamt Sachsen

La plupart sont proches de la frontière polonaise ou tchèque. Johanngeorgenstadt dans les Métallifères en est un exemple frappant. Avec une population divisée par deux, c'est une des villes les plus "rétrécissantes» (schrumpfende ou shrinking) d'Allemagne. La ville avait considérablement grossi grâce à l'extraction de l'uranium, passant de 6600 (1946) à 40000 h. en 1953. Le gisement épuisé, la population a chuté à 10600 (1960). D'autres activités industrielles se sont alors installées et une partie de l'habitat minier a été convertie en station de vacances mais cette base économique s'est effondrée après 1990. Depuis, divers projets pilotes ont été financés par le gouvernement et le Land: programmes Entwicklungs- und Sanierungsgebiete («zones de développement et de 
réaménagement ») en 2000 et Umbau von Siedlungsstrukturen unter Schrumpfungsbedingungen (" reconversion des immeubles résidentiels en contraction ») en 2005. Il était vain d'en espérer un succès durable comme le constatent Steinführer et Kabisch (2007): "La situation périphérique, la récession économique, le rétrécissement démographique, des facultés de réadaptation hors d'atteinte ainsi que l'image de soi d'une position "en marge" de la société et "sans avenir " forment un amalgame sinistre véhiculant une autoreprésentation de Johanngeorgenstadt qui se manifeste par un désespoir général ».

Par son évolution plutôt favorable, Bad Schlema (4 800 h.) illustre le type opposé de la petite ville revitalisée malgré une longue tradition minière typique des Monts Métallifères. Après-guerre, l'extraction intensive d'uranium a causé de tels affaissements que le centre et la station thermale devaient être démolis. L'exploitation ayant cessé après 1990, le maire a eu l'idée de réactiver la station thermale. La persévérance et les compétences des acteurs locaux expliquent la réussite de la stratégie de développement endogène soutenue par l'UE, le gouvernement et le Land et la participation des citoyens. La station a rouvert en 1998 et demeure l'activité principale de la ville.

L'évolution des petites villes bretonnes a été très différente.

\section{Les petites villes bretonnes : évolution et typologie}

\section{Une croissance supérieure à la moyenne régionale}

En France, l'image de la petite ville renvoie fréquemment au " pôle isolé » de l'INSEE, centre de services faisant fonction de lieu central. La littérature française entrevoit facilement leur "crépuscule » (Pumain, 1999; Roques, 2009) en raison des menaces pesant sur elles (déclin industriel, métropolisation, retrait des services publics, sélectivité des réseaux de transport) (Courcelle et al., 2017 ; Depraz, 2017). Or, le profil dominant de la petite ville française ou bretonne n'est plus celui-là. Les petites villes se rencontrent partout, même si leur nombre est fonction de la densité de population et du dynamisme démographique (figure 6). 
Figure 6. Les petites villes de Bretagne : population et évolution démographique (1990-2015).

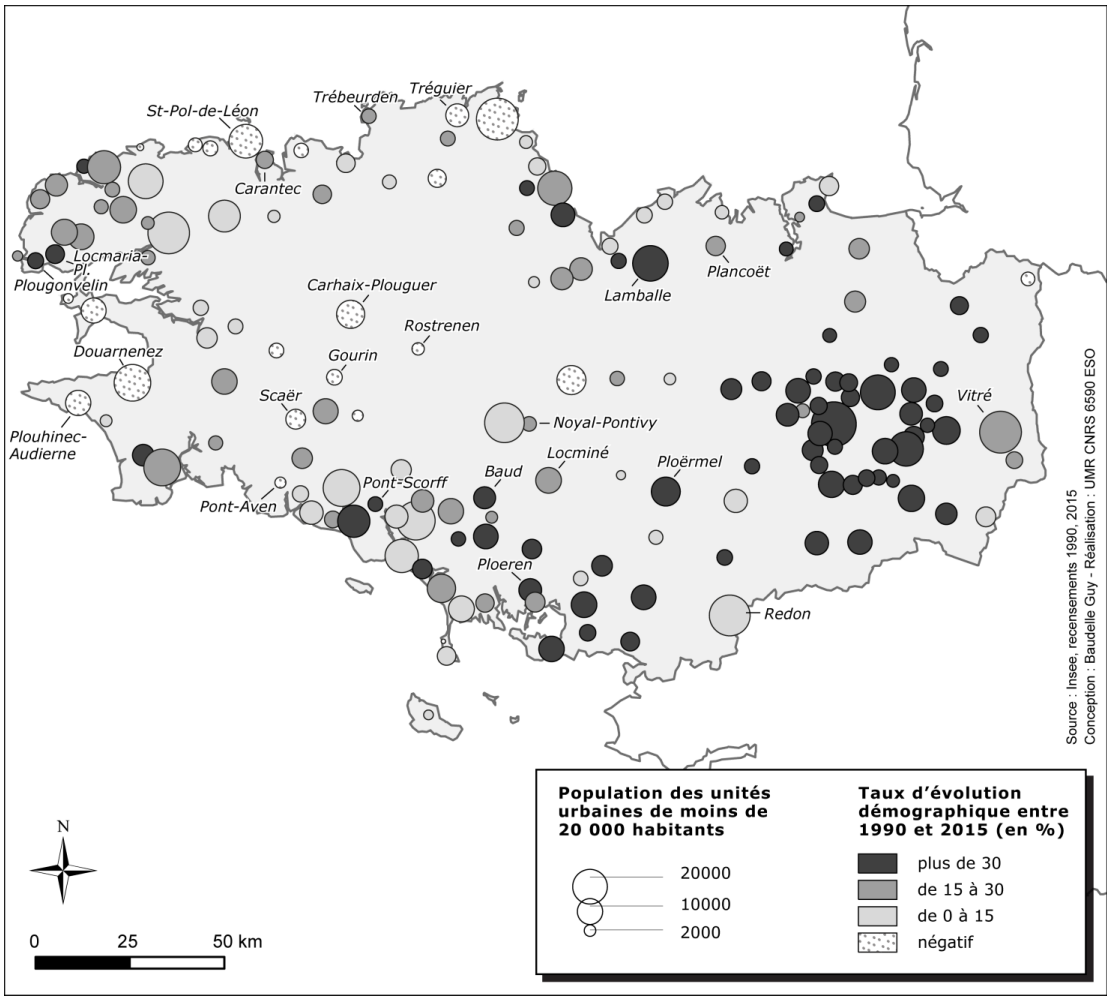

34 Les statistiques ne signalent pas de crise particulière des petites villes bretonnes dont la population a même augmenté à un rythme $(23,9 \%)$ supérieur à celui de la région $(17,8 \%)$, avec une croissance plus vive pour $59 \%$ d'entre elles. Elles ont presque toutes gagné de la population. Cette strate est également la plus dynamique au niveau national (Clanché, Rascol, 2011). En Bretagne, seule une vingtaine (soit $14 \%$ de l'échantillon) a subi une perte, rarement supérieure à $10 \%$ (tableau 5).

Tableau 5. Les petites villes bretonnes en fort déclin démographique (plus de $10 \%$ ) (1990-2015).

\begin{tabular}{lccc}
\hline Ville et département & 1990 & 2015 & Evolution en \% \\
\hline Louvigné-du-Désert (Ille-et-Vilaine) & 4260 & 3417 & $-19,8$ \\
Gourin (Morbihan) & 4734 & 3972 & $-16,1$ \\
Plouhinec-Audierne (Finistère) & 9181 & 7719 & $-15,9$ \\
Rostrenen (Côtes d'Armor) & 3664 & 3101 & $-15,4$ \\
Douarnenez (Finistère) & 16457 & 14208 & $-13,7$ \\
Plougasnou (Finistère) & 4191 & 3635 & $-13,3$ \\
Camaret-sur-Mer (Finistère) & 2933 & 2583 & $-11,9$ \\
\hline
\end{tabular}

Source : INSEE

Les situations sont toutefois très contrastées puisque inversement une dizaine d'entre elles ont vu leur population croître de plus des deux tiers, certaines faisant même plus que doubler leurs effectifs (tableau 6). 
Tableau 6. Les petites villes bretonnes en forte croissance démographique (plus de $90 \%$ ) (1990-2015).

\begin{tabular}{lccc}
\hline Ville et département & 1990 & 2015 & Evolution en \% \\
\hline Ploeren (Morbihan) & 2709 & 6611 & +144 \\
Châteaugiron (Ille-et-Vilaine) & 5673 & 12746 & $+124,7$ \\
La Mézière (Ille-et-Vilaine) & 2142 & 4644 & $+116,8$ \\
Gévezé (Ille-et-Vilaine) & 2434 & 5161 & +112 \\
Surzur (Morbihan) & 2081 & 4224 & +103 \\
Bourgbarré (Ille-et-Vilaine) & 2004 & 3841 & $+91,7$ \\
La Chapelle des Fougeretz (IIle-et-Vilaine) & 2513 & 4806 & $+91,3$ \\
Saint-Brice-en-Coglès (IIle-et-Vilaine) & 2484 & 4719 & +90
\end{tabular}

Source : INSEE locale de type proto-industriel (forges, textile) aux XVI ${ }^{\mathrm{e}}$-XVIII ${ }^{\mathrm{e}} \mathrm{s}$, avant l'effondrement industriel et démographique d'après 1860 (Bariou, 1974; Ollivro, 2005). Tourisme balnéaire d'abord puis, après-guerre, décentralisations industrielles et développement agro-alimentaire endogène ont permis de réactiver un certain nombre de petits centres.

\section{L'évolution contrastée des petites villes bretonnes : six profils}

Le profil actuel des petites localités est cependant beaucoup moins industriel qu'en Saxe. On peut distinguer six cas de figure en fonction du rythme d'évolution démographique depuis 1990 et des fonctions exercées par ces villes (tableau 7).

Tableau 7. Typologie des petites villes bretonnes d'après leur évolution démographique (1990-2015).

\begin{tabular}{|l|c|c|c|c|}
\hline Type & Nombre & $\begin{array}{c}\text { Part des petites } \\
\text { villes }\end{array}$ & $\begin{array}{c}\text { Taux de croissance } \\
1990-2015\end{array}$ & $\begin{array}{c}\text { Evolution } \\
\text { démographique } \\
\text { moyenne }\end{array}$ \\
\hline $\begin{array}{l}\text { Petites villes périurbaines en très } \\
\text { forte croissance }\end{array}$ & 54 & $34 \%$ & $>35 \%$ & $+63 \%$ \\
\hline $\begin{array}{l}\text { Autres localités périurbaines } \\
\text { dynamiques }\end{array}$ & 37 & $23 \%$ & de 18 à $35 \%$ & $+20 \%$ \\
\hline Petits pôles dynamiques & 11 & $7 \%$ & $\begin{array}{c}\text { de } 18 \text { à } 35 \% \text { (hors La } \\
\text { Gacilly) }\end{array}$ & $+21 \%$ \\
\hline $\begin{array}{l}\text { Petits lieux centraux faiblement } \\
\text { dynamiques }\end{array}$ & 25 & $10 \%$ & de 0 à $18 \%$ & $+9 \%$ \\
\hline Petites villes littorales de retraite & 20 & $12 \%$ & de 0 à $18 \%$ & $+8 \%$ \\
\hline Petits pôles en déclin & 22 & $14 \%$ & négatif & $-8 \%$ \\
\hline Ensemble des petites villes & 169 & $100 \%$ & $+25,8 \%$ & $+25,8 \%$ \\
\hline
\end{tabular}

Source : INSEE

Les petites villes périurbaines en très forte croissance (près du double du rythme régional) sont situées dans l'aire de déversement résidentiel proche de quatre agglomérations 
dynamiques (carte 2): Rennes - unité urbaine de $331000 \mathrm{~h}$. (2015) -, deux villes moyennes de la côte Sud - Vannes (79000 h.) et Auray (29000 h.) - et, avec moins d'intensité, Saint-Malo (46 000 h.). A elles seules, les aires urbaines de Rennes et Vannes ont absorbé la moitié du croît démographique total des 30 dernières années (Baudequin, 2014). Toutes les croissances record leur correspondent (tableau 5).

Les autres localités périurbaines dynamiques ont connu un taux de croissance supérieur à la moyenne régionale mais globalement trois fois moins vif. Ce type se divise en deux sous-catégories quasi égales :

- une quinzaine de localités périurbaines des aires urbaines dynamiques autour des mêmes quatre agglomérations mais généralement à plus grande distance (plus de $30 \mathrm{mn}$ ) (tableau 7);

- une vingtaine de localités périurbaines (sub)littorales de villes moyennes côtières (Lorient, Saint-Brieuc et Quimper) et de Brest (figures 7 et 8).

Figure 7. Plougonvelin, localité périurbaine et littorale à vive croissance.

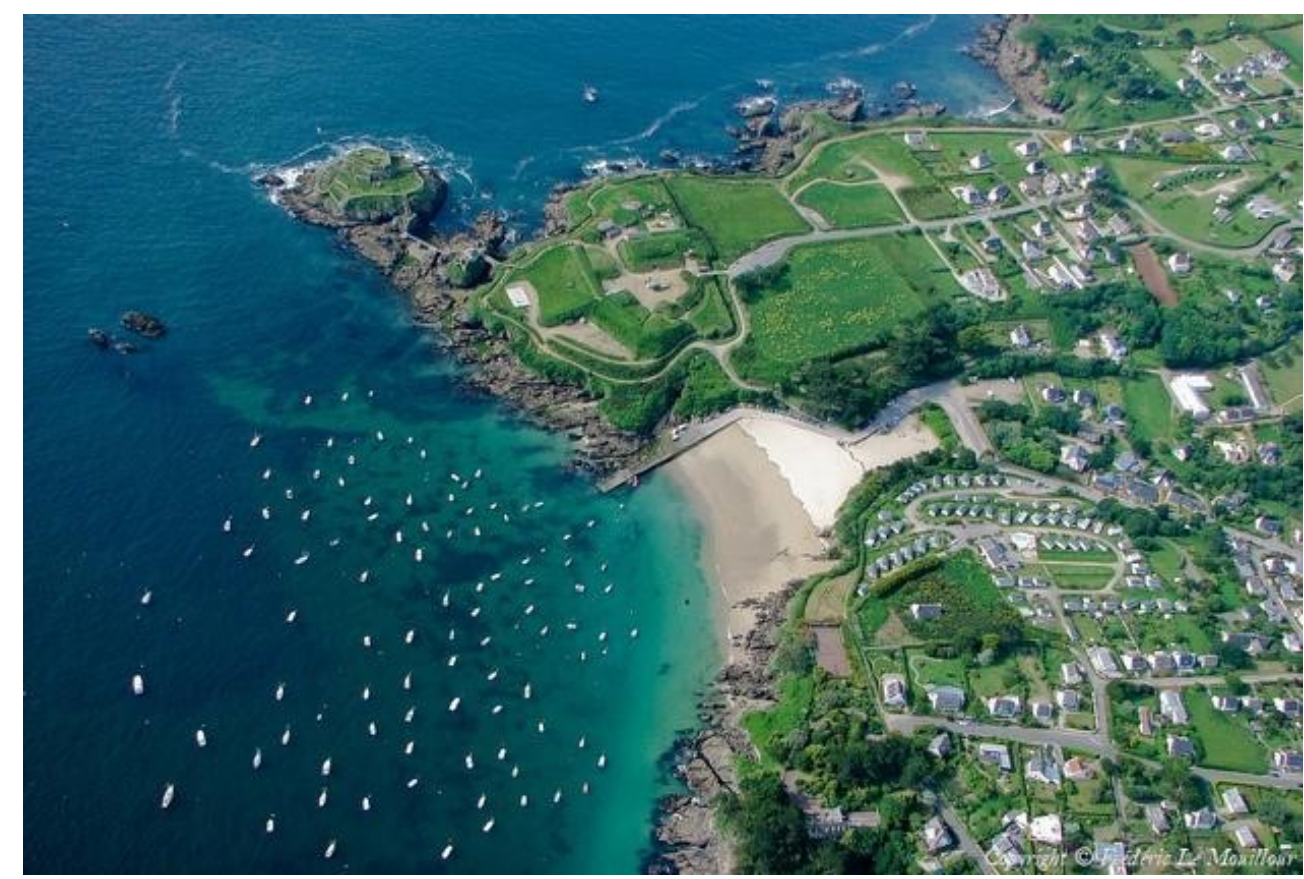

Source : http://iliketowastemytime.com/2012/12/16/aerial-france-36-pics 
Figure 8. Intensité du développement des lotissements à Guidel (près de Lorient), passée de 8200 à 11400 h. (+ $38 \%)$.

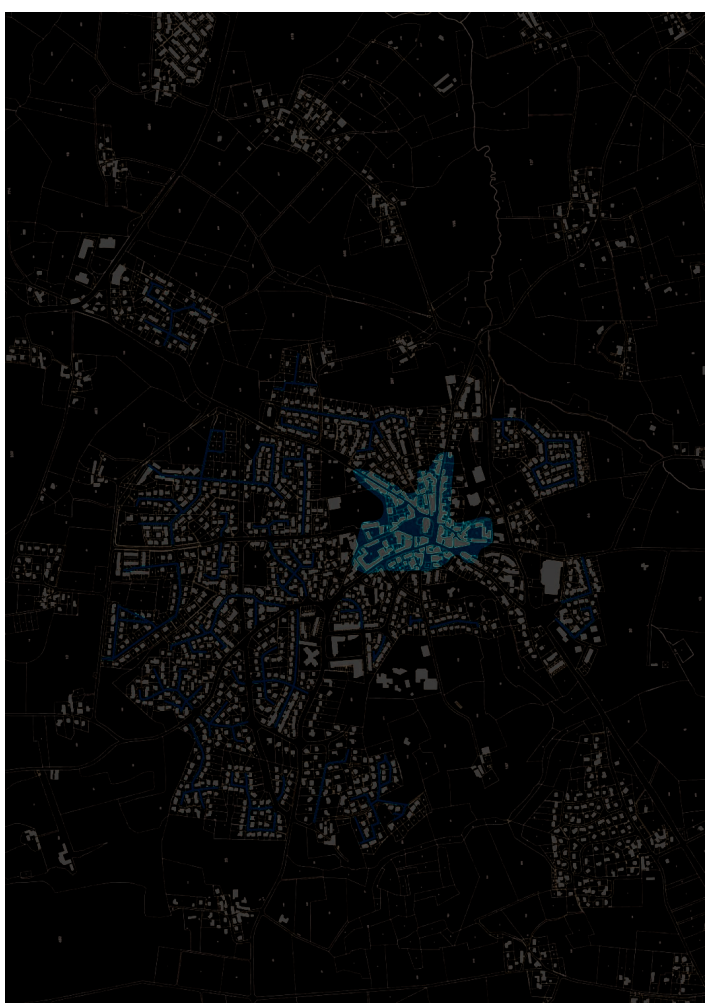

En rouge : le centre-bourg; lignes rouges : les nouvelles rues en extension urbaine

Source : CAUE (Conseil d'architecture d'urbanisme et d'environnement) 56 (2011), Atlas des paysages du Morbihan, Vannes

40 Ainsi, la moitié des petites villes bretonnes sont des localités périurbaines en croissance, pour partie littorales. La corrélation des taux de croissance avec la distance à la mer évaluée en distance-temps par la route $(0,32)$ ou en kilomètres $(0,39)$ est significative (figure 9).

Figure 9. Corrélation entre croissance des petites villes et proximité du littoral (en distance-temps par la route).

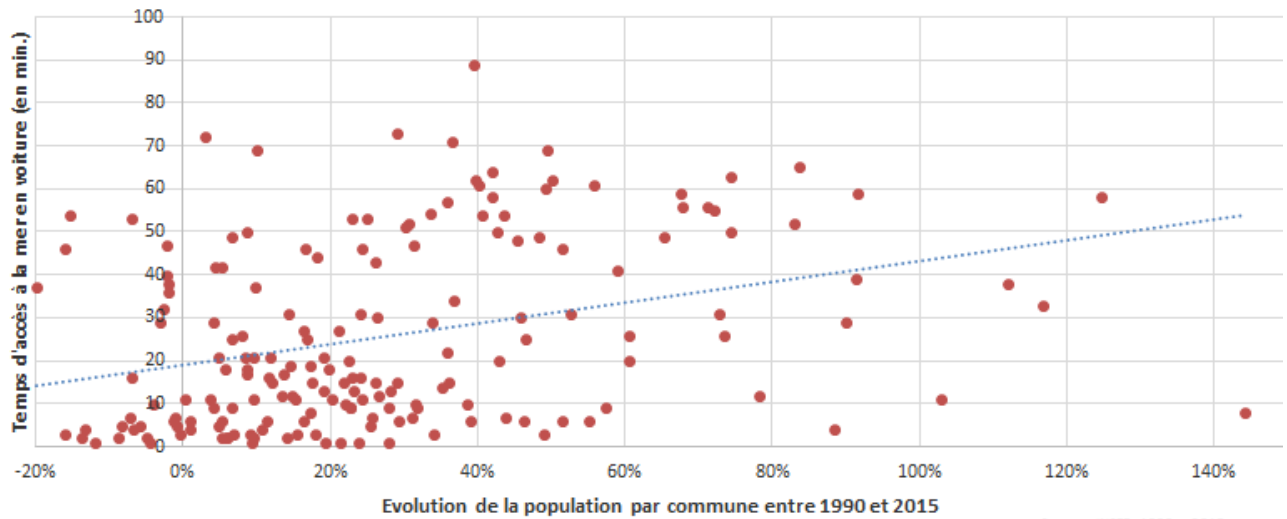

Source : INSEE ; données GPS 2018

41 En revanche, la proximité kilométrique d'une des 23 agglomérations principales de Bretagne ne semble pas influer sur ce taux $(-0,02)$ (figure 10$)^{2}$. Cette apparente 
neutralité illustre l'éloignement croissant de l'installation des ménages périurbains, la hausse étant plus vive dans la seconde couronne (entre 20 et $30 \mathrm{mn}$ de trajet) que dans la première (tableau 8).

Figure 10. Variation de la population des petites villes bretonnes en fonction de la distance à l'agglomération structurante la plus proche $(\mathrm{km})$.

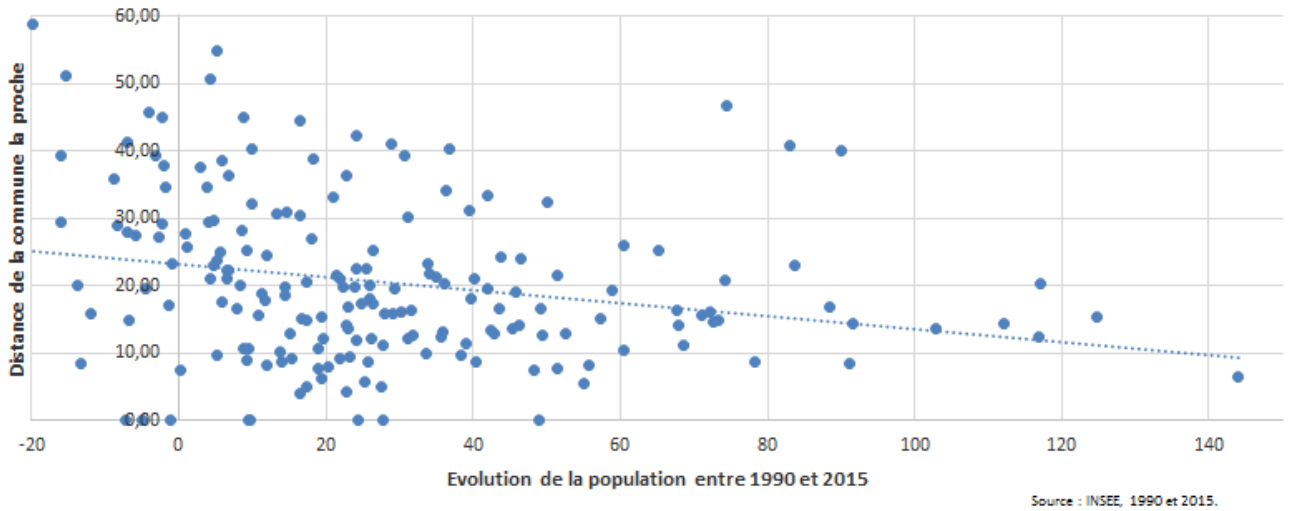

Source : INSEE

Tableau 8. Taux de croissance (\%) des unités urbaines (UU) selon le temps de trajet à l'agglomération structurante la plus proche.

\begin{tabular}{|l|r|r|}
\hline UU situées à moins de $10^{\prime}$ & 23 & $11,1 \%$ \\
\hline UU situées à moins de $20^{\prime}$ & 80 & $29,5 \%$ \\
\hline UU situées entre $20^{\prime}$ et $30^{\prime}$ & 70 & $33,3 \%$ \\
\hline UU situées à moins de 30' & 141 & $31,4 \%$ \\
\hline UU situées à plus de 30' & 39 & $12,5 \%$ \\
\hline
\end{tabular}

Le dynamisme n'est toutefois pas leur apanage dans la mesure où une douzaine de petits pôles dynamiques font même mieux en termes de croissance. La majeure partie correspond à des centres de production prospères où l'industrie pèse plus lourd dans l'emploi qu'en moyenne nationale (25\%) (Ambiaud et al., 2006). L'expansion du système agro-industriel de la première région agricole de France, notamment pour l'élevage, explique le développement de Vitré, Lamballe, Plancoët, Locminé ou Baud. Le tissu industriel est parfois plus diversifié (plasturgie, mécanique, chimie, bois). Contrairement à une représentation courante, industrie manufacturière et population ouvrière sont devenus le lot des petites villes (Servillo et al., 2014; Baudelle, Fache, 2015). Le meilleur exemple est celui de Vitré (encadré 1).

\section{Encadré 1 : Vitré, petite ville industrielle prospère bénéficiant d'un effet} d'emprunt

Bien placée sur l'axe Rennes-Paris, Vitré (18 000 h.) a su impulser une industrie alimentaire, automobile et électronique. En 40 ans, sa population a augmenté de près d'un tiers (près de deux fois plus vite que la population française) tout comme 
l'emploi industriel (+ $14 \%$ dans l'aire urbaine en dix ans contre une baisse de $10 \%$ au niveau national) et l'emploi privé ( $+7,5 \%$ contre $1,5 \%$ en France). La base productive par habitant $(32 \%)^{3}$ détient le record des 220 Communautés d'agglomération françaises. Le taux d'emploi de sa zone d'emploi $(74,6 \%)$ est près de deux fois supérieur à la moyenne nationale $(41 \%)$ et le taux de chômage $(5,1 \%$ au $1^{\mathrm{e}}$ trimestre 2018) le $4^{\mathrm{e}}$ plus faible du pays. Le développement endogène se double d'un effet de débordement depuis Rennes située à une demi-heure et probablement d'économies d'agglomération indirectes qualifiées d'effet de «taille empruntée » (borrowed size) par Alonso (1973) (Meijers et. al., 2016).

Les autres petits pôles dynamiques ont une économie présentielle (Talandier, Jousseaume, 2013): centres de villégiature littorale très attractifs (Carantec, Trébeurden sur la Côte de granite rose) ou localités résidentielles proches de petites villes moyennes (Morlaix, Tréguier).

Les petits lieux centraux faiblement dynamiques ont un taux d'évolution deux fois plus faible que la moyenne régionale. La dissymétrie géographique est très forte avec une «Bretagne à deux vitesses » opposant le Sud-est dynamique sur le plan démographique et économique au Nord-ouest. Bien que située au sud-est, Redon (18 000 h.) illustre ce type, avec une croissance modeste ( $4 \%$ ) et une forte vacance immobilière malgré les efforts de requalification (figure 11).

Figure 11. Redon : stagnation démographique et vacance immobilière malgré la rénovation du centre-ville.

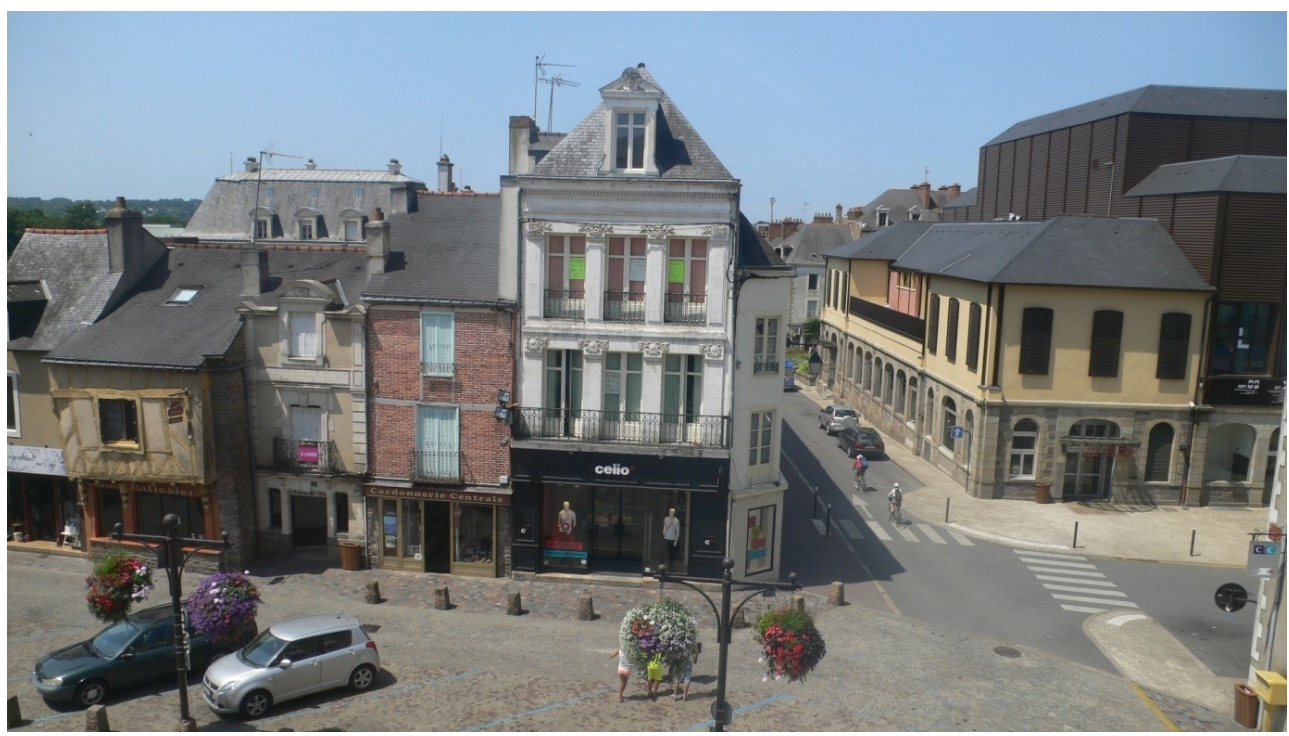

Source : ( ) G. Baudelle

Les petits pôles en déclin sont de même quasiment tous localisés à l'ouest d'une ligne Lorient-Saint-Brieuc, confirmant l'effet de périphéricité. La régression reste modérée mais contraste avec la santé globale des petites villes bretonnes. Deux sous-types se différencient par leur localisation : intérieure ou littorale.

Le sous-type classique est celui des lieux centraux intérieurs de campagnes vieillies et dépeuplées, déclinantes de longue date. Cette dizaine de centres de l'Arcoat ne représente qu'une petite ville sur dix mais la littérature les concernant est abondante 
car ils symbolisent le déclin quasi inexorable du Centre-Bretagne. Gourin (tableau 4) souffre ainsi d'un constant affaissement démographique typique d'une périphérie intérieure frappée par le vieillissement (recul de $39 \%$ par rapport au maximum de l'après-guerre : $6400 \mathrm{~h}$.). Son déclin a été accéléré par la fermeture de ses ardoisières (1962). L'effectif est ramené à son niveau de 1841. Rostrenen, Scaër ou CarhaixPlouguer sont d'autres exemples caractéristiques de la perte de substance de l'intérieur.

La sous-catégorie des petites villes littorales de retraite est plus inattendue. Il s'agit principalement de lieux de villégiature accueillant des retraités, si bien que le solde migratoire y est positif mais le solde naturel négatif. Ces communes résidentielles sont éloignées des agglomérations importantes, et moins attractives pour des raisons climatiques (côte nord) et/ou d'éloignement (Finistère). Pont-Aven, pourtant mondialement connue, figure dans cette classe. Les aléas des activités traditionnelles n'aident pas, qu'il s'agisse de la pêche (Douarnenez, Plouhinec-Audierne) ou des primeurs (Saint-Pol-de-Léon).

\section{Conclusion : I'hétérogénéité des trajectoires démographiques des petites villes}

Alors que les petites villes passent ordinairement pour avoir «le blues» (Erickcek, McKinney, 2006) compte tenu de perspectives réputées médiocres, leur dynamique est en réalité hétérogène comme l'indiquent les typologies dressées. C'est l'un des apports d'une étude régionale comparée qui, sans prétendre à l'extrapolation à l'échelle européenne, permet de confirmer les conclusions d'autres travaux (Burdack, Kriszan, 2013; Servillo et al., 2014; Delmas, Royoux, 2019), à commencer par l'extrême dépendance aux tendances démographiques régionales : "tant vont les régions tant vont leurs villes petites et moyennes. Hors des zones métropolitaines élargies, les dynamiques des villes petites et moyennes demeurent très sensibles à celles de leur région d'appartenance " (Cattan, Saint-Julien, 1999). L'évolution démographique générale, le solde migratoire et la structure par âge commandent très fortement les dynamiques (âge élevé en Saxe et dans certaines villégiatures littorales bretonnes, jeunesse du périurbain accueillant de nouvelles familles). L'évolution démographique est d'abord le reflet du contexte régional (Baron et al., 2010 ; Jousseaume, Talandier, 2016; Wolff, 2018). Cela s'explique en partie par le fait que les petites villes s'inscrivent dans des systèmes urbains dont les différentes composantes sont en interaction (Pumain, 2004), notamment à l'échelle régionale (Berroir et al., 2017).

Se trouve aussi confirmée la sensibilité aux dynamiques démographiques nationales: les difficultés des petites villes saxonnes sont à l'image de la démographie de l'Allemagne tout comme le dynamisme des bretonnes illustre celui de la France, comme l'ont montré d'autres études de cas ou analyses comparées (Burdack, Kriszan, 2013 ; Juan, Kunzmann, 2013). Consécutivement on note l'absence d'effet de taille vue l'hétérogénéité des trajectoires des petites villes (Servillo et al., 2014).

De même, le rôle capital de la périurbanisation autour des agglomérations dynamiques est bien un facteur de dynamisation, l'effet d'entrainement diminuant au-delà d'un certain seuil (Dubuc, 2004). A l'échelle européenne, les petites villes placées dans l'orbite d'agglomérations en développement représentent le cas de figure dominant (Wolff, 
2018). Peter Hall (2012) fait ainsi de la petite ville satellite le type majeur en Europe. Si les petites villes souffrent en un sens d'être "dans l'ombre de la métropolisation » sur le plan économique (Depraz, 2017), elles profitent en revanche à plein de la portée des métropoles sur le plan migratoire et démographique (Jousseaume, 1998). Le cas saxon montre toutefois que l'appartenance à une aire urbaine métropolitaine ne garantit pas le dynamisme en contexte démographique déprimé.

Le rôle de la base économique et du profil d'activités dans la dynamique des petites villes non périurbaines (Servillo et al., 2013) est également manifeste comme l'indiquent aussi bien la restructuration des villes saxonnes que la dynamisation de villes bretonnes.

Enfin, on a montré la fonction persistante de lieu central christallérien des petites villes en dehors des aires urbaines, en interaction avec les campagnes environnantes pour leur devenir réciproque.

Nos exemples infirment toutefois trois lois ordinairement reçues. D'abord, nulle obligation d'être placé dans "des zones métropolitaines élargies» ou "directement dans l'orbite des grandes métropoles» (Cattan et Saint-Julien, 1999) ni a fortiori de leur être connecté (Juan et Kunzmann, 2013) pour connaître la croissance : des villes moyennes attractives connaissent aussi une expansion démographique et un étalement périurbain d'ampleur suffisante pour entraîner de petites villes dans leur sillage. Secondo, l' économie résidentielle ne suffit pas nécessairement à assurer un gain démographique, les communes littorales bretonnes ayant connu une croissance plus faible $(+0,4 \%$ l'an) que les autres $(+1,1 \%)$ (Baudequin, 2014) en raison de leur vieillissement et des coûts fonciers ; ces facteurs fonciers qui expliquent aussi largement l'étalement périurbain et donc la croissance des petites villes proches des pôles dynamiques sont relativement minorés par la littérature (Servillo, 2014).

Dans l'ensemble, la distance aux agglomérations dynamiques, grandes ou moyennes, est donc un facteur explicatif très puissant des dynamiques des petites villes (Dubuc, 2004). Ce constat ne doit pas conduire à minorer les autres facteurs mentionnés ni d'autres éléments moins mécaniques susceptibles d'intervenir dans leur dynamique comme la gouvernance, l'aménagement, les effets d'apprentissage ou la capacité d'initiative (Fertner et al., 2015).

\section{BIBLIOGRAPHIE}

ALONSO W. (1973), « Urban zero population growth », Daedalus, 102, 4, pp. 191-206.

AMBIAUD É., BLANC M. \& SCHMITT B. (2006), « Les bassins de vie des bourgs et petites villes : une économie résidentielle et souvent industrielle », in INSEE, La France et ses régions, pp. 43-47, http://www.insee.fr/fr/ffc/docs_ffc/re f/fsr06e.pdf.

AUTHIER J.-Y., BIDOU-ZACHARIASEN C. (2017), « Editorial. Ces villes dont on ne parle pas », Espaces et sociétés, 1, 168-169, pp. 9-16, https://www.cairn.info/revue-espaces-et-societes-2017-1page-9.htm. 
BARIOU R. (1974), « Dépeuplement, exode, dépopulation en Bretagne centrale : l'exemple de la Haute-Cornouaille », Norois, 84, pp. 527-539.

BARLÖSIUS E., NEU C. (2008), « Territoriale Ungleichheit : eine spezifische Ausprägung räumlicher Untergleichheit », in ibidem (dir.), Peripherisierung - eine neue Form sozialer Ungleichheit ?, Berlin, Materialien der inderdisziplinären Arbeitsgruppe « Zukunftsorientierte Nutzung ländlischer Räume », 21, pp. 17-23.

BARON M. et al. (dir.) (2010), Villes et régions européennes en décroissance : maintenir la cohésion territoriale, Paris, Hermès \& Lavoisier, 345 p.

BATUNOVA E., GUNKO M. (2018), « Urban shrinkage : an unspoken challenge of spatial planning in Russian small and medium-sized cities », European spatial planning, 26, 8, pp. 1580-1597, https:// doi.org/10.1080/09654313.2018.1484891.

BAUDELLE G., FACHE J. (dir.) (2015), Les mutations des systèmes productifs en France, Rennes, PUR, $374 \mathrm{p}$.

BAUDEQUIN I. (2014), « Un demi-million de Bretons supplémentaires en 30 ans, dont $80 \%$ dans l'est de la région », Octant analyse, 56, 5 p., http://www.insee.fr/fr/themes/document.asp? reg_id=2\&ref_id=20644\#trois

BELL D., JAYNE M. (2009), « Small cities? Towards a research agenda », International journal of urban and regional research, 33, 3, pp. 683-699.

BERROIR S. et al. (2017), « Les systèmes urbains français : une approche relationnelle », cybergeo : European journal of geography [En ligne], document 807, mis en ligne le 6 février 2017, consulté le 16 décembre 2018, doi : 10.4000/cybergeo.27945.

BMVBS (Bundesministerium für Verkehr, Bau und Stadt-entwicklung) (dir.) (2013), Kleinere Städte und Gemeinden - überörtliche Zusammenarbeit und Netzwerke. Programmstrategie zum Städtebauförderungsprogramm, Berlin, Bonn, 28 p., https://www.staedtebaufoerderung.info/ StBauF/SharedDocs/Publikationen/StBauF/KleinereStaedte/Programmstrategie.pdf? _-_blob=publicationFile\&v=3.

BORSIG A., BURDACK J. \& KNAPPE E. (dir.) (2010), « Small towns in Eastern Europe : local networks and urban development », Beiträge zur regionalen Geographie, 64, 104 p.

BOSE M., WIRTH P. (2006), « Gesundschrumpfen oder Ausbluten? », Aus Politik und Zeitgeschichte, 21-22, pp. 18-24.

BURDACK J. (2010), « Small town development and social capital in Eastern Germany: the cases of Colditz and Leisnig ", Beiträge zur regionalen Geographie, 64, pp. 26-45.

BURDACK J., KRISZAN A. (dir.) (2013), Kleinstädte in Mittel- und Osteuropa : Perspektiven und Strategien lokaler Entwicklung, Leipzig, Leibniz-Institut für Länderkunde, Forum IfL19, 113 p., http://nbnresolving.de/urn:nbn:de:0168-ssoar-338681.

CANCHÉ F., RASCOL O. (2011), « Le découpage en unités urbaines de 2010. L'espace urbain augmente de $19 \%$ en une décennie », Insee première, 1364, http://www.insee.fr/fr/themes/ document.asp?ref_id=ip1364.

CARLOW V.M. (dir.) (2017), Ruralism. The future of villages and small towns in an urbanizing world, Berlin, Jovis, $296 \mathrm{p}$.

CATTAN N., SAINT-JULIEN T. (1999), « Quels atouts pour les petites et moyennes villes en Europe occidentale? », L’Information géographique, 63, 4, pp. 158-164, doi : 10.3406/ingeo.1999.2655, 
http://www.persee.fr/web/revues/home/prescript/article/

ingeo_0020-0093_1999_num_63_4_2655.

CHATEL C., MORICONI-EBRARD F. (dir.) (2016-2017), « Petites villes du monde », Territoire en mouvement [En ligne], 32 | 2016 et 33 / 2017, mis en ligne le 27 mars 2017.

CLANCHÉ F., RASCOL O. (2011), « Le découpage en unités urbaines de 2010 », INSEE première, 1364, 4 p., https://www.insee.fr/fr/statistiques/1280970.

COURCELLE T., FIYALKOW Y. \& TAULELLE F. (dir.) (2017), Services publics et territoires : adaptations, innovations et réactions, Rennes, PUR, 253 p.

DELMAS A., ROYOUX D. (2019, à paraître), « Les petites villes, un rôle clé dans la structuration urbaine de la Nouvelle Aquitaine », in CHAUCHEFOIN P. (dir.), Nouvelles régions : la grande transformation?, Rennes, PUR.

DEMAZIERE C. (2017), « Le traitement des petites et moyennes villes par les études urbaines », Espaces et sociétés, 168-169, pp. 17-32.

DEPRAZ S. (2017), « Marges industrielles et petites villes dans l'ombre de la métropolisation », ch. 7, in ibidem, La France des marges, Paris, A. Colin, pp. 193-222.

DUBUC S. (2004), « Dynamisme rural : l'effet des petites villes », L'espace géographique, 33, 1, pp. 69-85, doi : 10.3917/eg.033.0069, https://www.cairn.info/revue-espacegeographique-2004-1.htm-page-69.htm.

ERICKCEK G.A., MCKINNEY H. (2006), « Small cities blues: looking for growth factors in small and medium-sized cities », Economic development quarterly, 20, pp. 232-258.

FERTNER C. et al. (2015), « Small towns resisting urban decay through residential attractiveness: findings from Denmark », Geografisk tidsskrift/Danish journal of geography, 115, 2, pp. 119-132, https://doi.org/10.1080/00167223.2015.1060863.

GÁBOR P., TRÓCSÁNY A. \& MAKKAI B. (2015), « Between shrinking and blooming : the crossroad of small towns' urbanisation in Hungary ", Annales universitatis paedagogicae cracoviensis studia geographica, 8, pp. 12-28.

GRÖTZBACH E. (1963), « Geographische Untersuchung über die Kleinstadt der Gegenwart in Süddeutschland », Münchner geographische Hefte, 24, 106.

GRUNFELDER J., RISPLING L. \& NORLEN G. (dir.) (2018), State of the Nordic region 2018. Theme 1: demography, Copenhaguen, Nordic Council of Ministers, $59 \mathrm{p}$.

HALL P. (2012), « Balancing European territory. The challenge of the post-neoliberal era », in GANSER R., PIRO R. (dir.), Parallel patterns of shrinking cities and urban growth. Spatial planning for sustainable development of city regions and rural areas, Farnham, Ashgate, pp. 27-45.

HANNEMANN C. (2004), « Urbanistische Probleme und kulturelle Perspektiven der ostdeutschen Kleinstadtentwicklung », in ZIMMERMANN C. (dir.), Kleinstädt in der Moderne, 31, Stuttgart, Thorbecke, pp. 157-182.

JOUSSEAUME V. (1998), L'ombre d'une métropole : les bourgs-centres de la Loire-Atlantique, Rennes, PUR, $212 \mathrm{p}$.

JOUSSEAUME V., TALANDIER M. (2016), « Bourgs-centres et petites villes en France », Territoires en mouvement [En ligne], 32 | 2016, mis en ligne le 18 novembre 2016, http://

journals.openedition.org/tem/3887, doi : 10.4000/tem.3887. 
JUAN L., KUNZMANN K.R. (dir.) (2013), « Small and medium-sized cities: approaches to balanced development in Europe », $\mathrm{n}^{\circ}$ thématique, Urban planning international (国际城市规划), 28, 5, pp. 1-44.

KAMIŃSKA W., MULARCZYK M. (2014), « Demographic types of small cities in Poland », Miscellanea geographica : regional studies on development, 18, 4, pp. 24-33.

KAYSER B. et al. (1972), Les petites villes françaises, Toulouse, Travaux et documents du CIEU.

LABORIE J.-P. (1979), Les petites villes, Paris, Editions du CNRS, 363 p.

LEBER N., KUNZMANN K.R. (2006), « Entwicklungsperspektiven ländlicher Räume in Zeiten des Metropolenfiebers », DisP, 166, 3, pp. 58-70.

LENTZ S. (2004), « Klein- und Mittelstädte : der doppelte Wiederaufbau von Grimma, Wurzen und Eilenburg », Petermanns geographische Mitteilungen, 148, pp. 44-49.

LÜTTISCH H. (2017), Perspektiven deutscher Städte. Eine Clusteranalyse, Hünxe, Contor, 83 p., https:// www.thema-standortanalyse.de/e-books-zur-standortanalyse/perspektiven-deutscherst \%C3 \%A4dte/studie-als-pdf-dokument/.

MANFRED K. (2015), « Small towns in peripheral regions of Germany », Annales universitatis paedagogicae cracoviensis studia geographica, 8, pp. 29-38.

MARETZKE S. (dir.) (2018), Kleinere Städte und Gemeinden - überörtliche Zusammenarbeit und Netzwerke, Zweiter Statusbericht zum Städtebauförderungsprogramm, Bonn, BBSR, 72 p., https:// www.bbsr.bund.de/BBSR/DE/Veroeffentlichungen/Sonderveroeffentlichungen/2018/kleinerestaedte-gemeinden-dl.pdf.

MEIJERS E., BURGER M.J. \& HOOGERBRUGGE M. (2016), « Borrowing size in networks of cities: city size, network connectivity and metropolitan functions in Europe ", Papers in regional science, 95, 1, pp. 181-198, doi : 10.1111/pirs.12181.

OLLIVRO J. (2005), Bretagne : 150 ans d'évolution démographique, Rennes, PUR, 366 p.

PUCA (Plan Urbanisme Construction Architecture) (2018), Consultation Popsu Territoires pour des projets de recherche-action, Paris, 12 p., http://www.urbanisme-puca.gouv.fr/consultation-popsuterritoires-pour-des-projets-de-a1396.html.

PUMAIN D. (1999), «Quel rôle pour les villes petites et moyennes des régions périphériques ? ", Revue de géographie alpine, 87, 2, pp. 167-184, doi : 10.3406/rga.1999.2950, http://www.persee.fr/ web/revues/home/prescript/article/rga_0035-1121_1999_num_87_2_2950.

PUMAIN D. (2004), «Scaling laws and urban systems », Santa Fe institute working paper, n $04-02-002,26$ p., https://sfi-edu.s3.amazonaws.com/sfi-edu/production/uploads/sfi-com/dev/ uploads/filer/23/4d/234dc29d-faf5-4715-bd7e-a418c8cc9e9a/04-02-002.pdf.

ROQUES J.-L. (2009), La fin des petites villes. Une modernité envahissante, Paris, L'Harmattan, 226 p.

SANTAMARIA F. (2012), « Les villes moyennes françaises et leur rôle en matière d'aménagement du territoire : vers de nouvelles perspectives ? », Norois [En ligne], $223 \mid 2012$, mis en ligne le 28 février 2014, doi : 10.4000/norois.4180, http://journals.openedition.org/norois/4180.

SERVILLO L. et al. (2014), TOWN: Small and medium sized towns in their functional territorial context, applied research 2013/1/23, rapport final, Louvain, Katholieke Universiteit Leuven, Luxembourg, ESPON, 67 p., http://www.espon.eu/export/sites/default/Documents/Projects/AppliedResearch/ TOWN/TOWN-DFR_3.7_2014.pdf. 
STEINFÜHRER A., KABISCH S.(2007), « Binnen und Außenimage von Johanngeorgenstadt », in WIRTH P., BOSE M. (dir.), Schrumpfung an der Peripherie. Ein Modellvorhaben und was Kommunen daraus lernen können, Munich, Oekom, pp. 107-123.

STEINFÜHRER A., VAISHAR A. \& ZAPLETALOVÁ J. (2016), « The small town in rural areas as an underresearched type of settlement », European countryside, 8, 4, pp. 322-332.

TALANDIER M., JOUSSEAUME V. (2013), « Les équipements du quotidien en France : un facteur d'attractivité résidentielle et de développement des territoires ? », Norois, 1, 226, pp. 7-23, doi : $10.4000 /$ norois.4525.

TAULELLE F. (2010), « La France des villes petites et moyennes », in CAILLY L., VANIER M. (dir.), La France : une géographie urbaine, Paris, Armand Colin, pp. 138-154.

VARNA G., ADAMS D. \& DOCHERTY I. (2018), « Development networks and urban growth in small cities », European urban and regional studies, https://doi.org/10.1177/0969776418802919.

WOLFF M. (2018), Uneven urban dynamics : the role of urban shrinkage and regrowth in Europe, Thèse, Dortmund, Université technique, 197 p., http://dx.doi.org/10.17877/DE290R-19066.

WOLFF M., WIECHMANN T. (2017), « Urban growth and decline: Europe's shrinking cities in a comparative perspective 1990-2010», European urban and regional studies, 25, 3, pp. 122-139, doi : 10.1177/0969776417694680.

\section{NOTES}

1. Parfois moins en Allemagne du fait du statut municipal (Stadtrecht) octroyé à certaines communes de moins de 2000 habitants.

2. Calcul des distances à la mer et aux centres-villes des 23 agglomérations avec l'outil correspondant de $\mathrm{QGIS}^{\circledR} 2.18$ à partir des centroïdes de polygone des communes sélectionnées et des temps d'accès motorisés estimés à partir d'un système GPS. Collecte effectuée les $1^{\text {er }}$ et 2 décembre 2018.

3. La base productive correspond à l'emploi « exportateur » de la théorie de la base (cf. PORTIER O. (réd.) (2016), Le profil de développement des agglomérations et métropoles françaises, Paris, Assemblée des Communautés de France \& Caisse des Dépôts, 120 p., http://www.adcf.org/files/ DOCS/profil_developpement_agglo_2016-web.pdf).

\section{RÉSUMÉS}

L'article vise à montrer la diversité des facteurs d'évolution des petites villes européennes à partir d'une étude comparée de deux régions périphériques dans leur espace national mais sujettes à des évolutions démographiques opposées : la Saxe et la Bretagne. Après avoir présenté les hypothèses et choix méthodologiques, une typologie des trajectoires démographiques de ces villes de 1990 à 2015 par région est dressée. Les enseignements par rapport à l'état du savoir actuel en sont tirés : loin d'une crise globale, l'évolution des petites localités est très dépendante de leur distance aux agglomérations dynamiques (grandes ou moyennes) et de la santé démographique de leur région. L'économie résidentielle ne suffit cependant pas à leur assurer un 
avenir, la base économique et leurs fonctions de pôle de service restant des déterminants majeurs.

The diversity of the factors commanding the evolution of small European cities is addressed from a comparative analysis of two peripheral regions within their national space but subject to opposite demographic trends: Saxony (Eastern Germany) and Brittany (France). The assumptions and the methodological choices are presented. The population dynamics of the towns from 1990 to 2015 exhibit various types for each region. The results are used to reassess the current state of knowledge: far from a general crisis, the evolution of small cities is very dependent on their distance from dynamic (large or medium) agglomerations and the demographic dynamics of their region.

\section{INDEX}

Mots-clés : petites villes, Saxe, Bretagne, étude comparée, facteurs d'évolution démographique Keywords : towns, small cities, Saxony, Brittany, population dynamics

\section{AUTEURS}

\section{GUY BAUDELLE}

UMR ESO 6590 CNRS, Université Rennes 2, guy.baudelle@univ-rennes2.fr

\section{JOACHIM BURDACK}

Leibniz-Institut für Länderkunde, Leipzig, j_burdack@ifl-leipzig.de

\section{LOUIS-THIBAULT BURON}

UMR ESO 6590 CNRS, Université Rennes 2, louis-thibault.buron@univ-rennes2.fr 